\title{
A fuzzy approach in the study of COVID-19 pandemic in Brazil
}

\author{
Elenice Weber Stiegelmeier ${ }^{1}$ (D) - Glaucia Maria Bressan ${ }^{1}$ (D)
}

Received: 6 December 2020 / Accepted: 29 March 2021 / Published online: 12 April 2021

(C) Sociedade Brasileira de Engenharia Biomedica 2021

\begin{abstract}
Purpose The COVID-19 pandemic has spread widely through the world. Most countries impose severe intervention measures to contain the spread of the virus, and this worldwide scenario has motivated the development of researches in all areas of knowledge. In this context, this paper presents a study about how intervention measures such as lockdown, partial lockdown, and no-lockdown help to impede the extent of the severe outbreak of COVID-19.

Methods Since mathematical models are used to describe population dynamics and the behavior of epidemics, this paper presents a fuzzy approach to describe the behavior of new daily cases of COVID-19 in Brazil based on the p-fuzzy dynamic systems, considering as input variables, the infected population and the environment action. The evaluated output variable is the level of infestation.

Results The results of a fuzzy model showed that intervention measures play a crucial role in determining the success of COVID19 eradication programs, while there is no vaccine available for all the population. The proposed fuzzy model was developed by posing intervention measures and the results showed that to consider partial-lockdown helped to slow down the transmission rates of COVID-19 in the population, however the total lockdown is more effective, while the vaccine is not available.

Conclusion Therefore, mathematical models consist of an effective tool to investigate the situation with intervention strategies and estimate the potential benefits and costs of different strategies. The fuzzy model proposed assists government decisionmaking in order to minimize the economic impacts caused by the pandemic.
\end{abstract}

Keywords P-fuzzy dynamic systems · Population dynamics · Covid-19 $\cdot$ Intervention measures

\section{Introduction}

The COVID-19 pandemic officially began in late 2019 on the Asian continent, in China, and has spread widely through the continent. Since the emergence of this infectious disease, it has been subsequently span to every continent of the world except Antarctica (Rodríguez-Morales et al. 2020).

In Brazil, the Ministry of Health confirmed the first case of coronavirus in São Paulo, in Feb. 26, 2020. The rapid spread of COVID-19 pandemic has motivated the development of research in this area, and several research activities have been

Glaucia Maria Bressan

glauciabressan@utfpr.edu.br

Elenice Weber Stiegelmeier

elenicew@utfpr.edu.br

1 Department of Mathematics, Universidade Tecnológica Federal do Paraná, Cornélio Procópio, PR 86300-000, Brazil conducted for better understanding the origin, functions, treatments, and preventions of this novel coronavirus (Acter et al. 2020). According to Grzybowski et al. (2020), it has demanded a quick response from governments in terms of planning contingency efforts that include the imposition of social isolation measures and an unprecedented increase in the availability of medical services. Rahaman et al. (2020) suggest socio-health policies to be imposed, such as quarantine, social distancing, and lockdown measures to cripple the transmission of the virus. The sooner such measures are implemented, the shorter will be the term of the endemic.

Epidemic models have been proposed in the literature to describe epidemic dynamics, especially the dynamics of the COVID-19 epidemic. Each modeling approach seeks to describe a set of variables of interest according to specific objectives. Recent models in the literature for the study of COVID-19 are extensions of the well-known SEIR (susceptible-exposed-infected-removed) proposed by $\mathrm{Li}$ and Muldowney (1995), such as López and Rodó (2020). Then, the mathematical modeling of epidemic dynamics can provide a valuable contribution. 
The recent paper Grzybowski et al. (2020) presents an epidemic model specially tailored for the study of the COVID-19 epidemics. The model allows the integrated study of epidemic control strategies. Authors present a case study with three prognostic scenarios for the first wave of the epidemic in the city of Manaus, the capital city of Amazonas state, Brazil. Results show that there are feasible control strategies that could substantially reduce the overload within reasonable time.

Ordinary differential equations are a powerful tool for mathematical modeling of population dynamics. By knowing the phenomenon under study, it is possible to choose the function that determines the variation in relation to the state of the variable. This fact limits the modeling of many phenomena, since the relationship between state variables and their variations is either subjective or partially known. This modeling is possible using $\mathrm{p}$-fuzzy systems, which incorporate subjective information in both variables and variations and their relationships with variables, making the models more applicable and realistic (Barros and Bassanezi 2006).

Santos and Bassanezi (2009) propose a p-fuzzy dynamic system to model the population dynamics of a species that considers factors represented by a defined and coupled to the one-dimensional p-fuzzy system Environmental Condition.

In the context of the use of mathematical models to describe population dynamics and the behavior of epidemics, the objective of this paper is to present a fuzzy approach to describe the behavior of new daily cases of COVID-19 in Brazil based on the p-fuzzy dynamic systems. The input variables considered are the infected population and the environment action; the output variable is the level of infestation.

Deterministic modeling requires that we have a good knowledge of the phenomenon to be modeled, that is, that we know how the state variables and their variations are related. This fact limits the modeling of many phenomena, since in most cases the relationship between state variables and their variations is either subjective or partially known. To avoid this problem, we approach a mathematical modeling of population dynamics that incorporate subjectivities through fuzzy systems.

This paper is organized as follows: In the "Methods" section, we describe preliminary concepts about p-fuzzy systems, present classical population models, such as Verhulst p-fuzzy mod$\mathrm{el}$, and describe the formulation of the fuzzy model considering measure interventions. In the "Results" section, numerical simulations have been presented to illustrate the analytical results. The "Discussion" section presents a critical analysis of the pfuzzy approach in the light of existing knowledge. Lastly, the conclusions have been given in the "Conclusion" section.

\section{Methods}

Dynamic systems are subdivided into two classes: continuous and discrete. Continuous dynamic systems consist of a system defined by derivatives, since they represent rates of continuous variation. Discrete dynamic systems evolve over time through an iterative process. Finally, p-fuzzy dynamic systems refer to an abbreviation of the term partially fuzzy, i.e., the directions field, partially known a priori; it is obtained through methodologies of fuzzy controllers (Barros and Bassanezi 2006). Since the defuzzification processes are present, the solution of a p-fuzzy system is crisp, that is, an accurate $x(t)$ value that represents the state variable at each time $t$. For the study of important concepts and definitions about fuzzy set theory, see Pedrycz and Gomide (1998).

Briefly, in the theory of fuzzy sets, the so-called membership degree refers to the extent to which it is possible for an element to belong to a set. Processing of fuzzy information is usually done through operations that aggregate, combine, and compare the membership degree of their elements. Basically, a system based on fuzzy rules has four components: an input processor (fuzzification), a collection of linguistic rules called a rule base, a fuzzy inference method (Mamdani or Sugeno), and an output processor (defuzzification). One of the most common rules based fuzzy systems is known as fuzzy controllers. The partially fuzzy systems or p-fuzzy systems use rules based fuzzy systems in order to relate variables and their variations (Barros and Bassanezi 2006).

Definition 1 (Barros and Bassanezi 2006). A p-fuzzy initial value problem is given by Eq. (1):

$$
\begin{aligned}
& \frac{d u}{d t}=F(t, u(t)) \\
& u(a)=u_{0^{\prime}}
\end{aligned}
$$

where $F:[a b] \times F(R) \rightarrow F(R), u_{0} \in F(R)$ and $\frac{d u}{d t}$ represents the variation rate of function $u$ depending on $t$. In addition, $F$ is partly known and described by a fuzzy rule base.

Discrete fuzzy modeling comes from fuzzy controllers. Therefore, a p-fuzzy discrete system is given by Eq. (2):

$$
\begin{gathered}
x_{t+1}=F\left(x_{t}\right) \\
x_{0}=x\left(t_{0}\right),
\end{gathered}
$$

where $F(x)=x+\Delta x$ such as $F: R \rightarrow R^{n}$ and $\Delta x$ is the defuzzificated output provided by the fuzzy controller. Thus, it is possible to generalize a discrete p-fuzzy system as difference equation given by Eq. (3):

$x_{t+1}-x_{t}=\Delta\left(x_{t}\right)$

where $\Delta x$ is the output of the fuzzy controller, whose structure can be seen in Fig. 1.

Variations in discrete systems are qualified for the time intervals in which successive changes in inputs occur. Some requirements on the rule base can facilitate the study of the balance of p-fuzzy systems, as follows:

(i) The fuzzy sets of the rule base must be fuzzy numbers. 


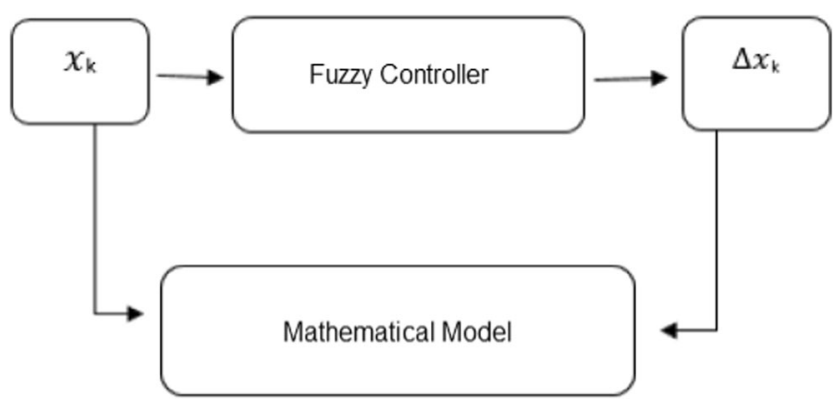

Fig. 1 P-fuzzy system general structure

(ii) The universes must be limited intervals from the set of real numbers.

(iii) Each element of the universe has non-zero membership to at least one of the fuzzy numbers in the rule base.

(iv) Each element of the universe has non-zero membership to a maximum of two antecedents.

(v) The elements of maximum membership (equal to 1) belong only to one of the numbers fuzzy of the rules base; that is, it has zero degree of membership to the other fuzzy numbers of the rules.

(vi) The rule base must be ordered monotonically (increasing or decreasing), so the qualifiers of the antecedents must be in ordinal scale, for example: small, medium, and large.

A rule base that satisfies these characteristics is called wellordered (Barros and Bassanezi 2006).

Depending on the rules used, the controller output field $f$ may present properties that guarantee the existence or uniqueness of the initial value problems' solution. However, depending on the complexity of $f$, it is necessary to use numerical methods to obtain estimates for the initial value problems' solution.

In this paper, the following were developed: the p-fuzzy system and the rules based fuzzy system associated, whose computational implementation was executed in the MATLAB application. Mamdani inference method was used as the fuzzy inference system, since fuzzy sets are desirable as outputs (Pedrycz and Gomide 1998), and the gravity center method was used as the defuzzification method (Pedrycz and Gomide 1998), both widely used in the literature.

\section{Classic population models}

There is a wide variety of biomathematical models in the literature, which consider the influence of some phenomena on the evolution of the population. In this section, the Verhulst logistic population model is presented to describe the population variation at time $t$, as well as a p-fuzzy approach for this model, which is used to model the behavior of the real data.

The Belgian mathematician Pierre Verhulst, around 1838, studied population models conditioned to limited resources.
Verhulst (1838) then presented the population growth equation as a differential equation, assuming that the population grows to a sustainable maximum limit, tending to stabilize and due to the limited resources. Let $a$ and $b$ be positive constants, the separable differential equation is given in Eq. (4):

$\frac{d p}{d t}=(a-b p) p$

Constant functions $p(t)=0$ e $p(t)=a / b \equiv k$ are equilibrium solutions of Eq. (1), since it refers to the case when there is no variation of $p$ when $t$ grows. Then, any equilibrium solution of Eq. (1) can be obtained by finding the roots of $f(p)=0$, and the zeros of $f(p)$ are the critical points. For $p_{0} \neq 0$ e $p_{0} \neq \frac{a}{b}$, the solution is given by Eq. (5):

$p(t)=\frac{a p_{0}}{b p_{0}+\left(a-b p_{0}\right) e^{-a t}}$.

If $t \rightarrow \infty$, then $p(t) \rightarrow k$; this value is called the limit population, and it is the asymptotic value of the population, for any initial population $p_{0}>0$. If $p_{0}>k$, the population $p(t) \mathrm{de}$ creases exponentially tending to $k$. If $0<p_{0}<k$, the population encreases tending to $k$. The tipping point is at $p(t)=\frac{a}{2 b}$; that is, until reaching the value $k / 2$, the population grows with a positive derivative, and from there the growth is gives more slowly.

\section{P-fuzzy model to describe the behavior of new daily cases of COVID-19 in Brazil}

Verhulst model can be considered a p-fuzzy model, considering some input variable as fuzzy. The objective is to find the stability of the model; therefore, it is necessary to present rules with semantic opposition, that is, alternation of signs in the consequent variations.

Let $x$ be the population and $\Delta(x)$ the variation rate, in the iteration $k$. The p-fuzzy system considered is given by Eq. (6):

$x_{k+1}=x_{k}+\Delta\left(x_{k}\right)$.

The input variable is the population, $x$, discretized into 6 classes: low, medium-low, medium, medium-high, high, and very high. The output is the variation rate, $\Delta\left(x_{k}\right)$, discretized into 4 classes: low-positive, low-negative, medium-positive, and high-positive. Thus, by making the semantic opposition in successive rules 5 and 6 , the following rule base was obtained:

1. If the population is low, then the variation is low-positive.

2. If the population is medium-low, then the variation is medium-positive.

3. If the population is medium, then the variation is highpositive.

4. If the population is medium-high, then the variation is medium-positive. 
5. If the population is high, then the variation is lowpositive.

6. If the population is very-high, then the variation is lownegative.

7. Using the rules base above, the membership functions are defined for the input and the output variables. Figure 2 shows the membership function for the input variable "infected population," corresponding the population, and Fig. 3 shows the membership function for the output variable "level-of-infected," representing the variation rate.

In this way, using numerical data provided by the Ministry of Health (Brazil), from February 26 (confirmation of the 1st case in Brazil) to August 4, 2020, a fuzzy approach to describe the behavior of new daily cases of COVID-19 in Brazil, using dynamic p- fuzzy model, is presented.

According to the literature (Pedrycz and Gomide 1998; Simões and Shaw 2007), fuzzy systems are specialist-type systems, that is, present behavior similar to human knowledge and operate in a specific domain. The membership functions are adjusted through empirical choices, based on specialist knowledge and on the function domain, to characterize inaccurate phenomena.

Figure 4 shows the data for new daily cases, represented by points, modeled by a dynamic p-fuzzy logistic model. The growth variation rate is the fuzzy parameter. Note that the solution of p-fuzzy model can be used to model the daily cases of COVID-19 in Brazil.

In the following subsection, we proceed to introduce the fuzzy model, the main aim of this paper, developed by posing intervention measures to contain the spread of COVID-19.

\section{Formulation of the fuzzy model}

As well known, the mathematical models are used to describe the complexity of biological phenomena, but there are always extrinsic factors that are determinant in the population dynamics and that cannot be modeled using classic deterministic

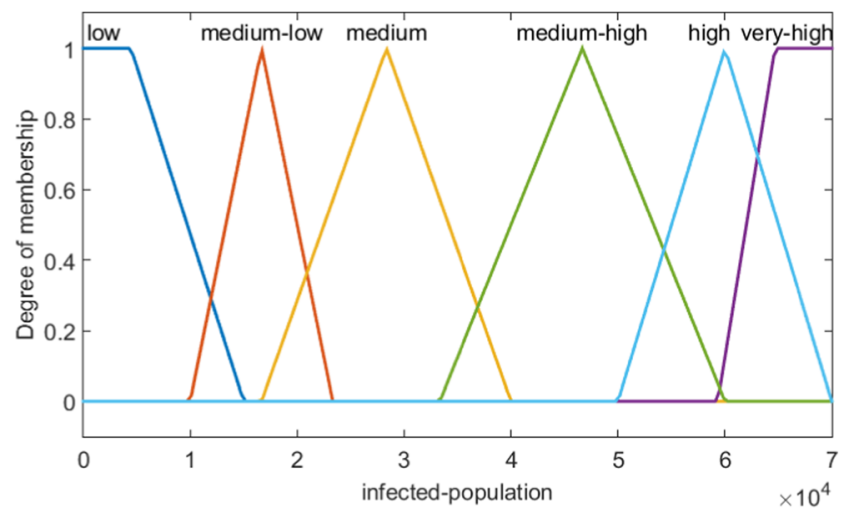

Fig. 2 Membership function for the input variable

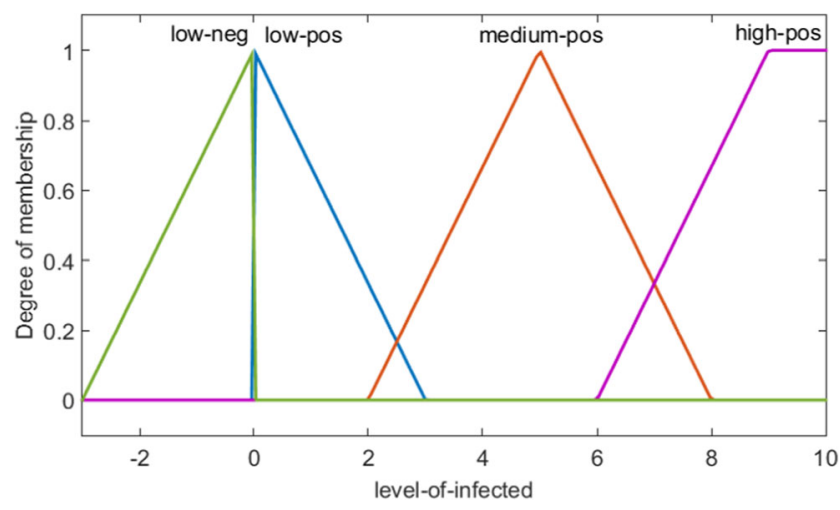

Fig. 3 Membership function for the output variable

model. Thus, to give a mathematical model the subjective topics, the fuzzy set theory, formalized by Zadeh (1965), is used. It can be, in many cases, an important tool for a better understanding some real situations, such as in dynamic populations.

In this paper, we consider the dynamic p-fuzzy logistic model, described in the "P-fuzzy model to describe the behavior of new daily cases of COVID-19 in Brazil" section, where the dynamic population is given by a fuzzy model. The proposed model below is developed by posing intervention measures to slow down the transmission rates in the population, while the vaccine is not available. We called these measures the environmental actions. Therefore, this study comprehends how intervention measures such as lockdown, partial lockdown, and no-lockdown help to prevent the extent of the severe outbreak of COVID-19.

We have aimed to observe the variation in the number of infected populations. In order to achieve this, we have considered the variables of the model number of population and environmental actions (inputs) and their variations (outputs).

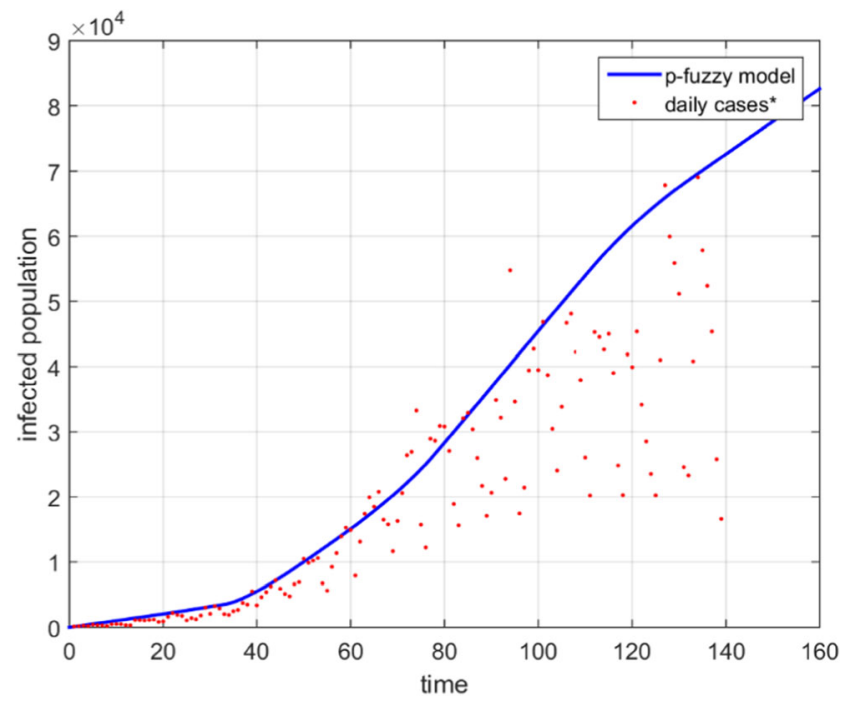

Fig. 4 Adjustment of the p-fuzzy logistic model with $p_{0}=100$. Source: Ministry of Health (2020) 
In this case, we utilized a rule based fuzzy system that gives the variation in function of the population and considers the period of time. However, accurate knowledge about the input variables and their variations is not available. On the other hand, qualitative information from specialists allows us to propose rules that relate to the variables of state, with their own variations.

The approach of this paper deals with a specific population type model, in which we simulate the dynamic of infected population by COVID-19 in face of intervention measures. In order to study this discrete p-fuzzy system, we consider that the input variables are the number of infected population $(\mathrm{x})$ and the intervention measures (y). The outputs represent the variation of infected populations. All the variables are linguistic, and each one of them can be described by terms such as low $(\mathrm{L})$, medium $(\mathrm{M})$, and high $(\mathrm{H})$ for the state variables, while high-positive $(\mathrm{H}+)$, medium-positive $(\mathrm{M}+)$, lowpositive $(\mathrm{L}+)$, low-negative $\left(\mathrm{L}^{-}\right)$, and high-negative $\left(\mathrm{H}^{-}\right)$are terms for the respective variations.

Suppose that such terms are modeled by fuzzy sets whose membership functions are obtained from a specialist. Such functions are represented in Fig. 6.

We make the following assumptions:

(i) To represent the subjectivity in the state variables, infected population density $(\mathrm{x})$, we discretized into 6 classes:

$I_{x}=\{$ low $(\mathrm{L})$, medium-low $(\mathrm{ML})$, medium $(\mathrm{M})$, medium-high $(\mathrm{MH})$, high $(\mathrm{H})$, and highest $(\mathrm{HH}\}$

For the variation of infected populations $\Delta(x)$, we considered the following:

$I_{\Delta(x)}=$ \{high-negative (H-), medium-negative (M-), low-negative(L-), low-positive (L+), medium-positive $(\mathrm{M}+)$, high-positive $(\mathrm{H}+)\}$

(ii) The variation of infected populations $\Delta(x)$ depends on the populational density of the specie; however, the increase (or decrease) of this rate will also be influenced by seasonality. That is, the species growth rate will change due to the intervention measures adopted.

(iii) There will be greater growth if no intervention measures are taken, and, when these are adopted, the birth rate decreases, reaching the point of having negative growth (only mortality) with a consequent decrease in the number of infected individuals.

(iv) The environmental actions (y) are represented by the set of linguistic variables:

$I_{y}=\{$ No-lockdown $(\mathrm{NL})$, medium-lockdown (ML) and total-lockdown (TL) $\}$

Thus, the environmental actions will contribute to degree the growth rate of the infected population.

(v) The input variable $y$ will depend on the stage of the interactive system (k).

As explained in item (i), the variation $\Delta(x)$ depends on the environmental actions that will be activated. (vi) We considered even if the infected population approaches to zero, they may increase again, provided the environment is favorable. That is, we considered that the virus has a latent state, and when the condition is favorable, the virus returned.

Hence, the proposed model has two input variables, the infected population density and the environment action in a determine period of time, and one output, variation of population $\Delta(x, y)$. Figure 5 shows the p-fuzzy model general structure used.

Figure 6 shows the membership functions of the inputs (infected population and environment action) and the output (level of infestation) variables.

We noticed that in Fig. 6b, the environmental actions has a dominium in $D(y)=[0,180]$, that is, $F: D \rightarrow[0,1]$. Thus, we considered a period of 180 iterations, where in the first 60 iterations, we obtain the membership degree in the nolockdown set, and after that in the medium-lockdown set, and, from 120 iterations, the total-lockdown linguistic variable is activated.

In this paper, we may find an explicit view of the time behavior of environmental actions as a cyclical system, since the virus behaves cyclically. The result is estimated according to Eq. (7):

$y(k)= \begin{cases}k=q & \text { if } k \in\{1 \ldots 180\} \\ k=180-q & \text { if } k \in\{181 \ldots 360\} \\ & \vdots \\ k=q & \text { if } k \in\{361 \ldots K\}\end{cases}$

where $q, k, \in, \mathbb{N}$ and $K$ are the number of iterations, based on Santos and Bassanezi (2009).

Following the assumptions (i)-(vi), the system fuzzy rule base is given by 18 rules of the type:

"IF the number of infected-population $\mathrm{x}$ is $A_{r}$ AND the environmental-actions y is $B_{r}$ THEN the variation of population $\Delta(x)$ is $C_{r}$."

where $A_{r} \in I_{x}, B_{r} \in I_{y}$ and $C_{r} \in I_{\Delta(x)}$ and $r \in\{1 \quad \ldots 18\}$ is the rule considered.

Table 1 shows the 18 linguistic rules constructed, the ifthen rules base. For example: "If $x$ is medium-low (ML) and $y$ is no-lockdown (NL), so the variation $\Delta(x)$ will be highpositive (H+)."

From the rules base presented in Table 1, we can infer that if there is a favorable condition for the virus, that is, we activate no-lockdown in the environment action, so the population growth rate is the highest; if the environment is always favorable, so the growth rate is positive, independent on the population size, and an unfavorable environment makes the population variation rate negative (mortality higher than birth). 
Fig. 5 P-fuzzy model structure considered

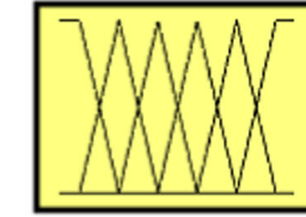

infected-population (6)

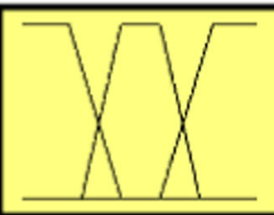

environment-actions (3)

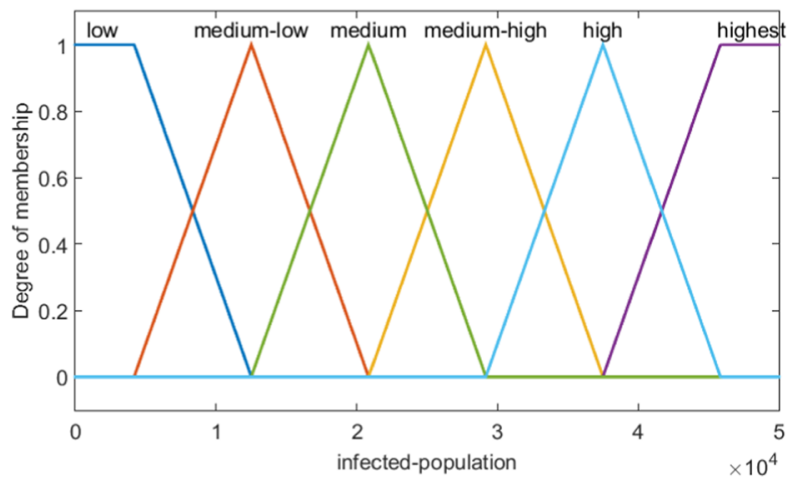

(a)

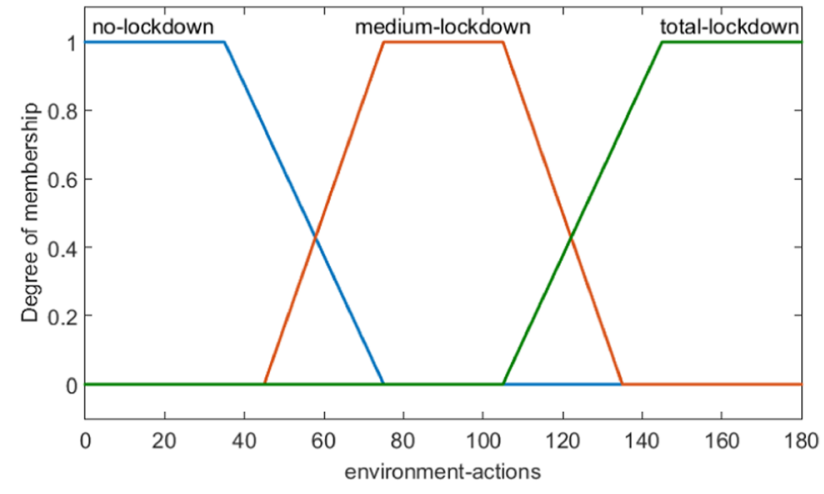

(b)

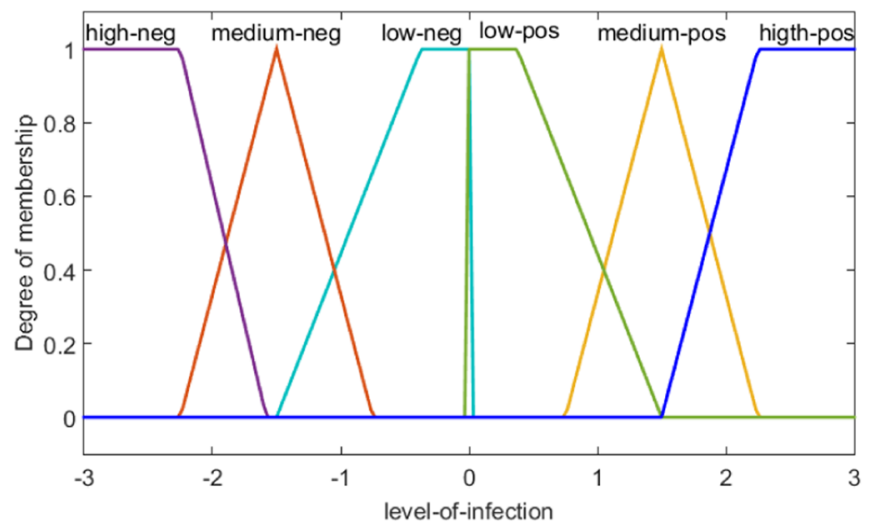

(c)

Fig. 6 Input variables: a infected population and $\mathbf{b}$ environment action. Output variable: $\mathbf{c}$ level of infestation 
Table 1 Rules base for the p-fuzzy model used in Eq. (8)

\begin{tabular}{llll}
\hline $\boldsymbol{x y}$ & $\begin{array}{l}\text { No- } \\
\text { lockdown }\end{array}$ & Medium lockdown & $\begin{array}{l}\text { Total- } \\
\text { lockdown }\end{array}$ \\
\hline Low & M+ & L+ & L- \\
Medium-low & H+ & M+ & L- \\
Medium & H+ & H+ & M- \\
Medium-high & H+ & M+ & M- \\
High & M+ & L+ & H- \\
Highest & L- & M- & H- \\
\hline
\end{tabular}

Therefore, the p-fuzzy model proposed is given by Eq. (8):

$\left\{\begin{array}{c}x_{k+1}=x_{k}+\Delta\left(x_{k}, y_{k}\right) \\ \left(x_{0}, y_{0}\right) \in \mathbb{R} \times[0,180] \rightarrow \mathbb{R}\end{array}\right.$ where $\Delta(x, y)$ is given by the rules base shown in Table 1 and $y$ is according to Eq. (7).

\section{Results}

In this section, the dynamical behavior of the system is investigated numerically. In the fuzzy model, analysis depends directly on those parameters which define the rates at which individuals move from one stage to another. The fuzzy logic toolbox presented in software MATLAB (Hanselman and Littlefield 2003) was used to implement the fuzzy model approached.

Algorithm 1 describes the main code used to solve the fuzzy used in the numeric simulation.

\footnotetext{
Algorithm 1 - The main code used to solve the fuzzy model proposed

Input: $x_{0}, y_{0}, K$

Output: infected-population

Choose initial values: $\boldsymbol{x}_{\mathbf{0}}, \boldsymbol{y}_{\mathbf{0}}, \boldsymbol{K}$

for $\mathrm{k}$ de 1 até $\mathrm{K}$ do

$\Delta_{x} \leftarrow F\left(x_{k}, y_{k}\right)$

$x_{k+1}=x_{k}+\Delta_{x}$

end

infected-population $=\boldsymbol{x}_{\boldsymbol{k}}$
}

The simulated was implemented using MATLAB (www. mathworks.com). All experiments were run on a $2.4 \mathrm{GHz}$ Intel core $\mathrm{i} 7$ processor $8 \mathrm{~Gb}$ of RAM memory and Windows 10 operating system. The average processing time is considered fast.

We take for all the simulation an initial infected population of 2000 daily cases, and the intervention measures were changed to analyze the impact of each measure adopted in the dynamic of population. We chose this initial population to demonstrate the effected by the intervention measures in the dynamics of the virus. The simulation range is 300 iteration that's the days period.

We evaluated the dynamics of the infected population considering the measure intervention like no-lockdown, partial lockdown, and total-lockdown. Here, the no-lockdown is when no intervention measure is adopted to contain the spread of the virus; partial-lockdown means that average intervention measure was adopted by the countries; and total-lockdown means social distancing measures taken to hinder the spread (Rahaman et al. 2020).

We define a scenario for the simulation where the initial condition of infected population and the period of time are fixed, $x_{0}=2000$ (daily cases) and $K=300$ iterations, respectively. The environmental action is varied to active each linguist variable in set $I_{y}$. The goal here is to demonstrate how the intervention measures could be contributed to contain the spread of virus.

Figure 7 illustrates the evaluated of the infected population dynamic considering the scenario where none intervention measure was adopted, so we activated the linguist variable no-lockdown. Note that in the simulation period, the infected population grow faster in the begin period. We observe that the population decrease over the time but return to grow when no-lockdown is active again. It is clear in Fig. 7 that the growth is bigger than the initial period, representing the second wave of virus.

In Fig. 8, we activate the linguistic variable partiallockdown. In this case, we observe a slowdown in the number of daily cases. However, there is a second wave around 100 days, since the linguistic variable nolockdown is active in second cycle and the model shows the third wave if no intervention measure was adopted around 270 days. 


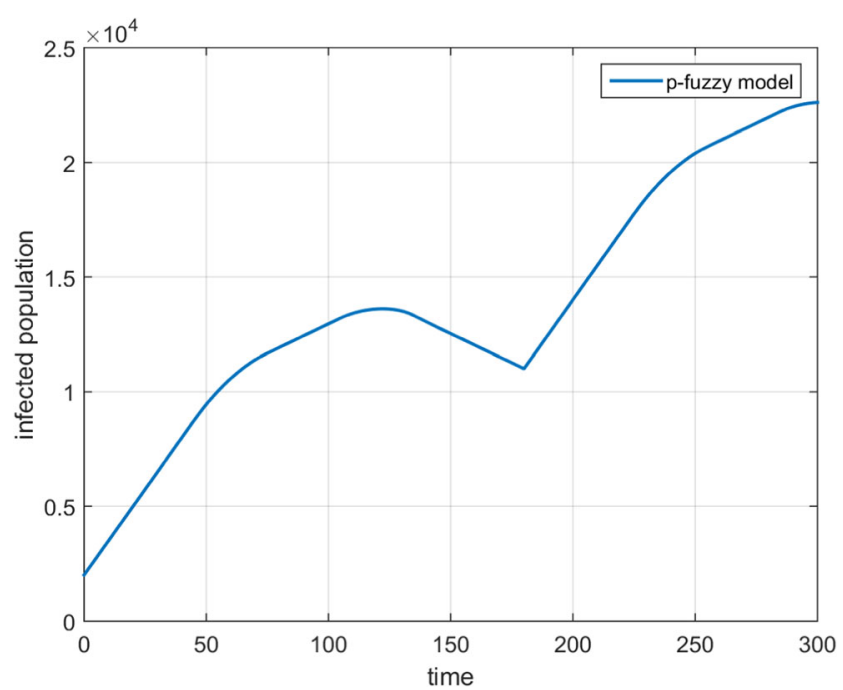

Fig. 7 P-fuzzy model solution with $y_{0}=0$, no-lockdown active in set $I_{y}$

Figure 9 illustrates the case where intervention measure strong, total-lockdown, is initially taken. We noticed that a drop occurs in the daily cases during the first days; it occurs due to the high level of intervention adopted (total-lockdown). However, if the population relaxed the measure, the virus returns at higher levels.

In Fig. 10, we show the output of the p-fuzzy system given by a surface, using the variable of the problem.

\section{Discussion}

Many proposed strategies for mitigating the spread of coronavirus has investigated the use of interventions (Rahaman et al. 2020; Aldila et al. 2020; Wang et al. 2020). The model proposed in this paper offers important insights in controlling the

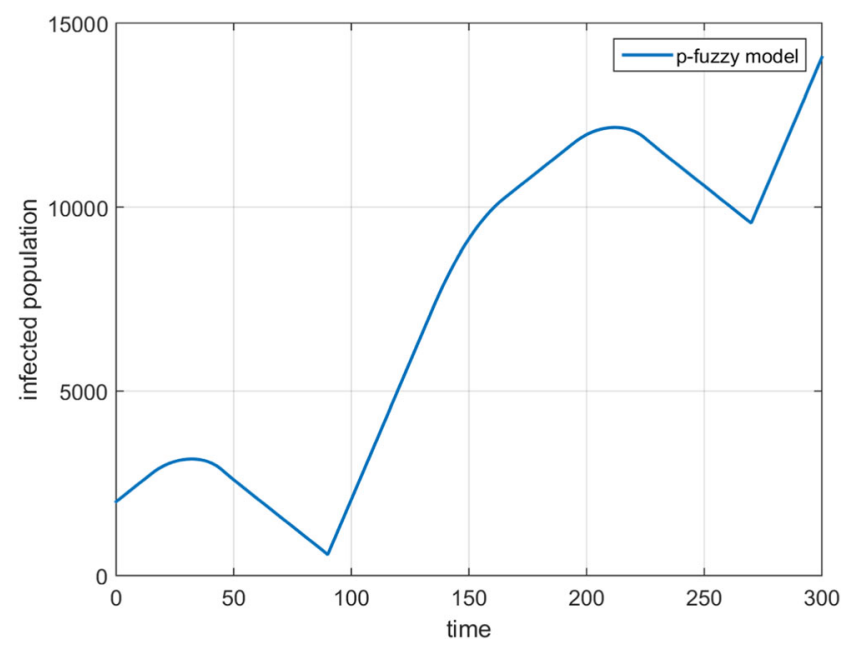

Fig. 8 P-fuzzy model solution with $y_{0}=90$, partial-lockdown is active in set $I_{y}$

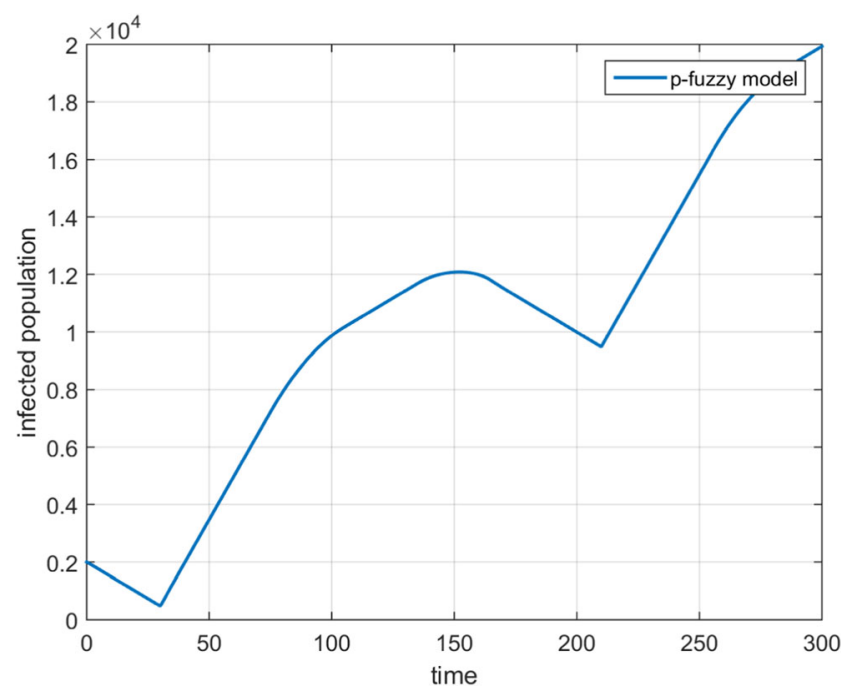

Fig. 9 P-fuzzy model solution with $y_{0}=150$, total-lockdown is active in set $I_{y}$

spread of COVID-19 using a p-fuzzy approach. The results of p-fuzzy model were effective since we get to understand that the intervention measures are the key to control the level of infected population. Thus, when fuzzy mathematical models are combined with the best intervention measure, the level of infected population can be mitigated.

This paper has suggested a fuzzy modeling for the evolution of infected population, focusing on the intervention measures and their effect in the increase of new cases of COVID19. The main difference between the classic model (4) and the fuzzy proposed model (8) is that the fuzzy model exploits parameter uncertainty, whereas the classic model does not. In this sense, the classic model can be considered a particular instance of fuzzy models. According to data from https:// coronavirus.jhu.edu/map.html, the second wave of the pandemic, or the second cycle of the virus, has been more aggressive than the first one, and the results presented in this paper demonstrate this fact, as can be seen in Figs. 7, 8, and 9.

According to Acter et al. (2020); Rahaman et al. (2020); and Aldila et al. (2020), among the possible preventive

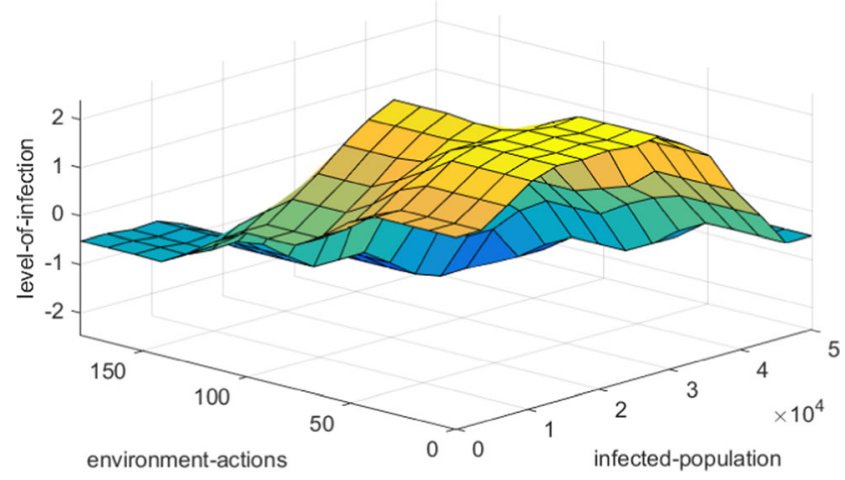

Fig. 10 The output of the p-fuzzy system illustrated by a surface 
measures, the lockdown is the most efficient strategy to contain the coronavirus spread. In addition, mathematical models (Grzybowski et al. 2020; López and Rodó 2020; Wang et al. 2020 ) indicate that the only way to stop the spread of COVID19 is the social isolation measures. In this sense, these results converge with the results presented by the fuzzy model proposed in this paper.

Therefore, the proposed fuzzy model contributes to minimize the risk of infection when social distancing was adopted. However, the implementation of these strategies can be more effective if there is a high level of individual awareness. If the government becomes aware of the importance of such interventions, they would decide to implement these measures. The present work could be used as an alternative solution to understand the population infected dynamics considering intervention measures.

\section{Conclusion}

Although a vaccine to protect the susceptible population from COVID-19 has not reached everyone, various interventions have been implemented by the government in many countries, such as social distancing and rapid testing, among others. In this work, we presented two mathematical models in the form of p-fuzzy systems to describe the dynamics of infected population and consider intervention measures to understand the dynamics of the coronavirus. The first model was constructed by modeling the daily cases of COVID-19 in Brazil through the fuzzy approach. The second model used a similar approach, but we added intervention measures to analyze the infected population dynamics.

Therefore, mathematical models consist of an effective tool to investigate the sophisticated situation with intervention strategies and estimate the potential benefits and costs of different strategies. The purpose of the paper to use fuzzy set theory in epidemiology, which is a recent area of research, was achieved. The proposed fuzzy model was developed by posing intervention measures and the results showed that to consider partial-lockdown helped to slow down the transmission rates of COVID-19 in the population, however the total lockdown is more effective, while the vaccine is not available for all the population. In addition, the fuzzy model assists government decisionmaking in order to minimize the economic impacts caused by the pandemic.

These prevention measures lead to a sudden decline in transmission rate of COVID-19 and turn out to be an effective strategy in containing the virus and saving lives.
Authors' contributions The authors contributed equally to the development of this work.

Data availability Not applicable.

Code availability Not applicable.

\section{Declarations}

Conflict of interest The authors declare no competing interests.

\section{References}

Acter T, Uddin N, Das J, Akhter A, Choudhury TR, Kim S. Evolution of severe acute respiratory syndrome coronavirus 2 (SARS-CoV-2) as coronavirus disease 2019 (COVID-19) pandemic: a global health emergency. Science of the Total Environment. 2020;730:138996.

Aldila, D.; Ndii, M. Z.; Samiadji B. M. Optimal control on COVID-19 eradication program in Indonesia under the effect of community awareness. Math Biosci Eng, vol. 17, n 6, p. 6355-6389, 2020.

Barros LC, Bassanezi R. Tópicos de lógica fuzzy e biomatemática. Textos didáticos. 2006;5. Campinas: IMECC.

BRAZIL. Ministry of Health (2020) Brazil https://covid.saude.gov.br

Grzybowski JMV, da Silva RV, Rafikov M. Expanded SEIRCQ Model Applied to COVID-19 Epidemic Control Strategy Design and Medical Infrastructure Planning. Mathematical Problems in Engineering. 2020:Article ID 819856315 pages, 2020.

Hanselman, D.; Littlefield, B. MATLAB 6-Complete Course. Prentice Hall, 2003.

Li MY, Muldowney JS. Global stability for the SEIR model in epidemiology. Math Biosci. 1995;125(2):155-64.

López L, Rodó X. A modified SEIR model to predict the COVID-19 outbreak in Spain and Italy: simulating control scenarios and multi-scale epidemics, Available at SSRN 3576802, 2020.

Pedrycz W, Gomide F. An introduction to fuzzy sets: analysis and design: MIT Press; 1998.

Rahaman MA, Islam MDS, Khan AA, Sarker B, Mumtaz A. Understanding "quarantine," "social distancing," and "lockdown" during "COVID-19" pandemic in response to global health: a conceptual review. Open J Soc Sci. 2020;8:283-305.

Rodríguez-Morales AJ, Macgregor K, Kanagarajah S, Patel D, Schlagenhauf P. Going global - travel and the 2019 novel coronavirus. Travel medicine and infectious disease. 2020;33:Article 101578. https://doi.org/10.1016/j.tmaid.2020.101578.

Santos LR, Bassanezi RC. Sistemas p-fuzzy unidimensionais com condição ambiental. Biomatemática. 2009;19:11-24.

Simões MG, Shaw IS. Controle e modelagem fuzzy. Blucher. 2007.

Verhulst PF. Notice sur la loi que la population suit dans son accroissement. In: Garnier JG, Quetelet A, editors. Correspondance Mathematique et Physique. Gand: H. Vandekerckhove fils; 1838, t. 10. p. 113-21.

Wang N, Fu Y, Zhang H, Shi H. An evaluation of mathematical models for the outbreak of COVID-19. Precision Clinical Medicine. 2020;3(2):85-93. https://doi.org/10.1093/pcmedi/pbaa016.

Zadeh LA. Fuzzy sets. Inf Control. 1965;8:338-53.

Publisher's note Springer Nature remains neutral with regard to jurisdictional claims in published maps and institutional affiliations. 